BMJ Open

Diabetes

Research

\& Care

\title{
Testing the sympathetic nervous system of the foot has a high predictive value for early amputation in patients with diabetes with a neuroischemic ulcer
}

\author{
Sanne M Schreuder, ${ }^{1}$ M Nieuwdorp, ${ }^{2}$ Marc J W Koelemay, ${ }^{3}$ Shandra Bipat, ${ }^{1}$ \\ Jim A Reekers ${ }^{1}$
}

To cite: Schreuder SM, Nieuwdorp M, Koelemay MJW, et al. Testing the sympathetic nervous system of the foot has a high predictive value for early amputation in patients with diabetes with a neuroischemic ulcer. BMJ Open Diab Res Care 2018;6:e000592. doi:10.1136/ bmjdrc-2018-000592

Received 6 August 2018 Revised 31 October 2018 Accepted 7 November 2018
Check for updates

\section{(C) Author(s) (or their} employer(s)) 2018. Re-use permitted under CC BY-NC. No commercial re-use. See rights and permissions. Published by BMJ.

${ }^{1}$ Department of Radiology and Nuclear Medicine, Amsterdam UMC, Amsterdam, The Netherlands

${ }^{2}$ Department of Vascular Medicine, Amsterdam UMC, Amsterdam, The Netherlands ${ }^{3}$ Department of Surgery, Amsterdam UMC, Amsterdam, The Netherlands

\section{Correspondence to} Professor Jim A Reekers; j.a.reekers@amc.uva.nl

\section{ABSTRACT}

Objective There is evidence from the literature that dysfunctionality of the sympathetic nervous system of the foot with subsequent loss of local autoregulation could be a predictor of early amputation in patients with diabetes with a neuroischemic ulcer. To confirm this we tested the functionality of the sympathetic nervous system in the foot in a consecutive group of 31 patients with diabetes with critical limb ischemia and non-healing neuroischemic ulcer.

Research design and methods Prospective cohort with retrospective analysis after 12 months of routinely acquired clinical data. All patients in the study group underwent angiography of the foot as part of a routine angioplasty procedure. Primary study endpoint was lower extremity amputation-free survival at 12 months. Because of the study design no other endpoints could be analyzed. The functionality of the sympathetic nervous system was tested with perfusion angiography.

Results Thirty-one patients were followed for 12 months. The Capillary Resistance Index (CRI) was used to measure the response of the sympathetic nervous system. $C R I \geq 0.9$ is the cut-off point for a non-responsive sympathetic nervous system. All patients $(n=11)$ with a $C R l \geq 0.9$ underwent a major amputation before 12 months. Of all patients with a CRI only $15 \%$ underwent major amputation. The positive predictive value for major amputation before 12 months for patients with a CRI $\geq 0.9$ was $100 \%$. Conclusions A non-responsive sympathetic nervous system of the foot is a strong predictor of early major amputation (log rank $\mathrm{p}<0.001$; HR 14.22; $95 \% \mathrm{Cl} 3.64$ to 55.51).

\section{INTRODUCTION}

Diabetes-related foot complications are the major cause of lower limb amputation. The presence of peripheral arterial disease (PAD) in particular is associated with an increased risk of ulceration, failure of ulcer to heal and amputation. ${ }^{1}$ Many parameters have been identified as possible predictors for poor outcome in patients with diabetic foot disease, for example, renal failure, dialysis, infection, gangrene and PAD. ${ }^{2} 3$ Prediction models for early amputation such as the

\section{Significance of this study}

What is already known about this subject?

- Over the past 20 years some publications have suggested that a dysfunctional sympathetic nervous system of the foot could be a predictor for poor outcome of neuroischemic ulcer in patients with diabetes. These papers have never attracted much attention.

\section{What are the new findings?}

- The inability to reduce the peripheral resistance in the foot with a local alpha-blocker is a predictor for a local dysfunctional sympathetic nervous system of the foot and amputation before 12 months.

- Perfusion angiography offers a very simple test to evaluate the functionality of the sympathetic nervous system by measuring change in total blood flow through the foot.

How might these results change the focus of research or clinical practice?

- The initial clinical impact of our findings could be that in patients with diabetes with a Capillary Resistance Index $(\mathrm{CRI}) \geq 0.9$, and a remaining or worsening non-healing ulcer after revascularization, a second intervention is probably in vain.

- Whereas in patients with a $\mathrm{CRl}<0.9$ a reintervention might be useful. This might be a cost-effective strategy, especially because this test does not add to the cost of the intervention.

Wound, Ischemia, and foot Infection classification have been introduced. ${ }^{4}$ Of all patients with diabetes who undergo successful endovascular or bypass revascularization, $>20 \%$ will still undergo major amputation within 12 months. ${ }^{5}{ }^{6}$ In $>50 \%$ off all amputations the revascularization is still patent at the time of amputation. ${ }^{6}$ This percentage has not decreased over the past decade, despite major advances in percutaneous revascularization technologies. ${ }^{78}$ As well as PAD, microangiopathy has been recognized as an 
important contributor to poor outcome in diabetic foot disease. Hemodynamic dysregulation with subsequent chronic capillary hypertension and associated high flow in the thoroughfare channels is recognized as one of the possible causes off microcirculation disease.$^{9-12}$ A functional sympathetic nervous system plays a crucial role in the normal hemodynamic autoregulation of the microcirculation. ${ }^{13} 14$ This study is based on several publications suggesting that dysfunctionality of the sympathetic nervous system of the foot is a strong predictor of early amputation in patients with diabetes with a neuroischemic ulcer. ${ }^{13-18}$

Until recently total blood flow in the foot could only be measured in the preclinical setting using either venous occlusion plethysmography or local isotope washout techniques. ${ }^{19}$ The new technique of perfusion angiography is able to instantly measure the total blood flow through the foot in the clinical setting. ${ }^{20}$ We used this new imaging technique to test the patients' variable of the functionality of the sympathetic nervous system in a consecutive group of patients with diabetes with neuroischemic ulcer and matched this with the main outcome in these patients, that is, amputation-free survival (AFS).

\section{MATERIALS AND METHODS \\ Patients}

From our routine angioplasty population we prospectively followed a consecutive group of 34 patients with diabetes with critical limb ischemia (CLI; toe pressure $<30 \mathrm{~mm}$ $\mathrm{Hg}$ ) and a non-healing neuroischemic ulcer. Neuroischemic ulcer is defined as neuropathy and vascular disease as an important contributor. A non-healing ulcer is defined as a wound that does not improve after 4 weeks or does not heal in 8 weeks. CLI was defined as an absolute toe pressure $<30 \mathrm{~mm} \mathrm{Hg}$ in patients with compressible vessels. Neuropathy was identified in this study when the treating diabetologist has made a note of this in the patients' medical record. Only patients with neuropathy were eligible for this study.

All patients were scheduled for angiography and subsequent angioplasty between April 2013 and January 2017. Three patients were lost to follow-up after the procedure and were excluded from analysis. A total of 31 patients were included for analysis. Of this group, 24 patients had type 2 diabetes mellitus and seven had type 1 diabetes. All patients were using insulin.

\section{Clinical management}

At our institute preintervention screening is done with Duplex/Doppler ultrasound only. Suitability for endovascular revascularization was determined during diagnostic angiography by an experienced interventional radiologist. All treated patients received 6 months of double antiplatelet treatment, and thereafter single antiplatelet medication. Secondary risk management and wound care were carried out in accordance with standard procedures at our hospital. In the clinical workup in our institution, diabetic ulcers are not always routinely scored with an ulcer classification system. Because this study was done based on the available routine data, a precise scoring of each patient is not available. In retrospect we could identify a mix of Wagner grades $1-4$. Patients with severe gangrene (Wagner 5) are in our institution not considered primary candidates for endovascular revascularization.

For this study we obtained a waiver from the local ethical committee, as all data were obtained as part of normal routine procedure and routine follow-up. No extra investigations or study-related follow-up appointments had taken place. Perfusion angiography is a postprocessing modality using standard digital subtraction angiographic (DSA) data.

\section{Technique}

The technique of perfusion angiography has been described before..$^{20}$ For postprocessing the DSA data we used the 2D perfusion software (Philips Healthcare, Best, The Netherlands). Briefly, with perfusion angiography the total flow (volume/time) through the foot, both the macrocirculation and microcirculation, is measured in a 2D region of interest. After injection of a standardized bolus of contrast the 2D perfusion software analyzed the change in density in each angiographic image voxel over time. To test the functionality of the sympathetic nervous system in the foot we blocked the alpha-adrenergic receptors of the arterioles locally using an alpha-receptor blocker (Tolazoline, Novartis, Basel, Switzerland). Four milligrams of Tolazoline (diluted in $10 \mathrm{~cm}^{3} \mathrm{NaCl} 0.9 \%$ ) was given through a 5 French end-hole catheter, with the tip in the mid-section of the popliteal artery. Blocking the alpha-adrenergic receptors results in instant dilatation of the arterioles, which only have alpha-adrenergic receptors, with direct lowering of the peripheral resistance and subsequent faster flow through the microcirculation. In patients with CLI the macrovessels will not dilate after administration of an alpha-blocker because these vessels are already maximally vasodilated by vasoactive metabolites released from ischemic tissue. On a graph faster total blood flow is represented as a curve with a lower peak, as passage of the contrast bolus is faster and subsequently there is less accumulation of contrast over time (figure 1).

A functional sympathetic nervous system is needed to elicit this response to Tolazoline. ${ }^{21}$ By comparing foot flow before and after local intra-arterial administration of Tolazoline, the response of the sympathetic nervous system can be tested. The response is measured as the Capillary Resistance Index (CRI), which is the peak of the flow post-Tolazoline divided by the peak pre-Tolazoline. ${ }^{20}$ This very low dose of Tolazoline has no systemic circulatory side effects. For more than 20 years Tolazoline in combination with angiography has routinely been used in our practice to obtain better flow and more optimal images in situations of local low flow. ${ }^{22}$ All perfusion angiography images were obtained under identical 

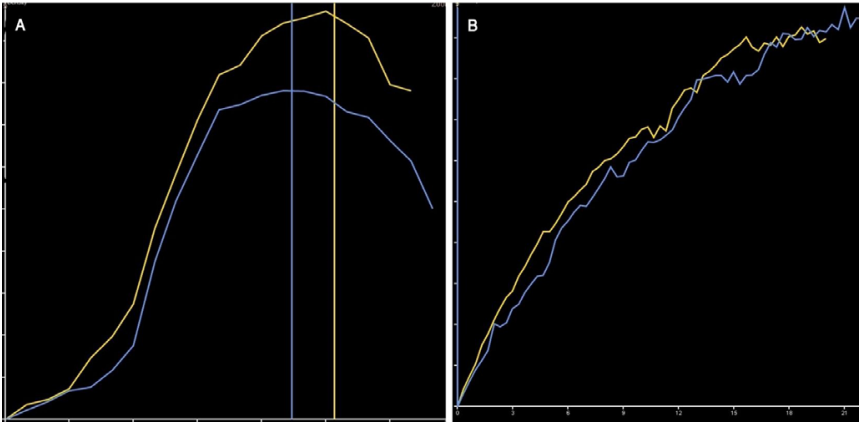

Figure 1 Time-density curves of a positive response and non-response to the Tolazoline administration. Yellow curve is foot perfusion before Tolazoline. Blue curve is foot perfusion after $4 \mathrm{mg}$ Tolazoline intra-arterial in mid-popliteal artery. (A) Normal time-density curves. First part parallel and early peak of the blue. $C R I=0.7$. (B) Non-responsive time-density curves. Curves overlap, no early peak density. $\mathrm{CRI}=1.0$. CRI, Capillary Resistance Index.

standardized conditions at both the start and the end of each procedure.

\section{Follow-up}

We analyzed patients after a period of 12 months or until time of death. Time of amputation or death was derived from medical records.

The perfusion data were stored on a secured local hard disk and the results were calculated independently from the clinical outcome at the end of follow-up. CRI was calculated at 12 months. The primary outcome was defined as lower extremity AFS. AFS is a composite endpoint of mortality and amputation and is defined as being alive without the need for a lower limb amputation. ${ }^{23}$ Amputation was defined as lower limb amputation, while minor amputations distal to the ankle were regarded as part of the treatment. There are important differences in minor amputation rates between the European centres, which can be explained in part by severity of disease at presentation. We therefore have decided to only report on major amputations because this has been analyzed and reported extensively in many studies and is recognized as a strong study endpoint in CLI studies. ${ }^{24}$

\section{Statistical analysis}

Baseline data were expressed as proportions (\%) and means \pm SD.

First, the cut-off for positive and negative response to alpha-blocking was defined by plotting the CRI values for both the amputee and non-amputee groups within 12 months. Second, we related the cut-off point for positive or negative response to alpha-blocking to AFS at 12 months. The sensitivity, specificity, positive predictive value (PPV) and negative predictive value (NPV) with $95 \%$ CIs were calculated using Altman. ${ }^{25}$

AFS was also compared in patients who underwent angioplasty and patients who did not undergo angioplasty. AFS was also analyzed in relation to type 1 or type

\begin{tabular}{ll}
\hline Table 1 & Baseline characteristics \\
\hline & $\begin{array}{l}\text { Total study } \\
\text { population }(\mathbf{n}=\mathbf{3 1})\end{array}$ \\
\hline Male, $\mathrm{n}(\%)$ & $21(68)$ \\
\hline Female, $\mathrm{n}(\%)$ & $10(32)$ \\
\hline Age (years) mean $\pm \mathrm{SD}$ & $68.9 \pm 12.9$ \\
Diabetes type 1, $\mathrm{n}(\%)^{*}$ & $7(23)$ \\
Diabetes type 2, $\mathrm{n}(\%)^{*}$ & $24(77)$ \\
\hline Patients with renal insufficiency or dialysis (\%) & $13(42)$ \\
\hline
\end{tabular}

${ }^{*}$ All patientsnsulin for their diabetes.

*All patients used insulin for their diabetes.

2 diabetes and dialysis or renal insufficiency. Finally, mortality was compared with CRI.

Time to event was estimated using Kaplan-Meier survival analysis and compared with log rank tests. In addition, HRs with 95\% CIs are given. Analyses were done with SPSS (V.23.0.0.2).

\section{RESULTS}

Our observational study sample comprised 31 patients with a mean age of $68.9( \pm 12.9 \mathrm{SD}), 21(68 \%)$ patients were male. The baseline characteristics of the patients are listed in table 1.

Twenty-three patients $(74 \%)$ were technically suitable for angioplasty and underwent the procedure successfully. In eight patients with the vessel anatomy below the knee, vessel anatomy was not suitable for endovascular

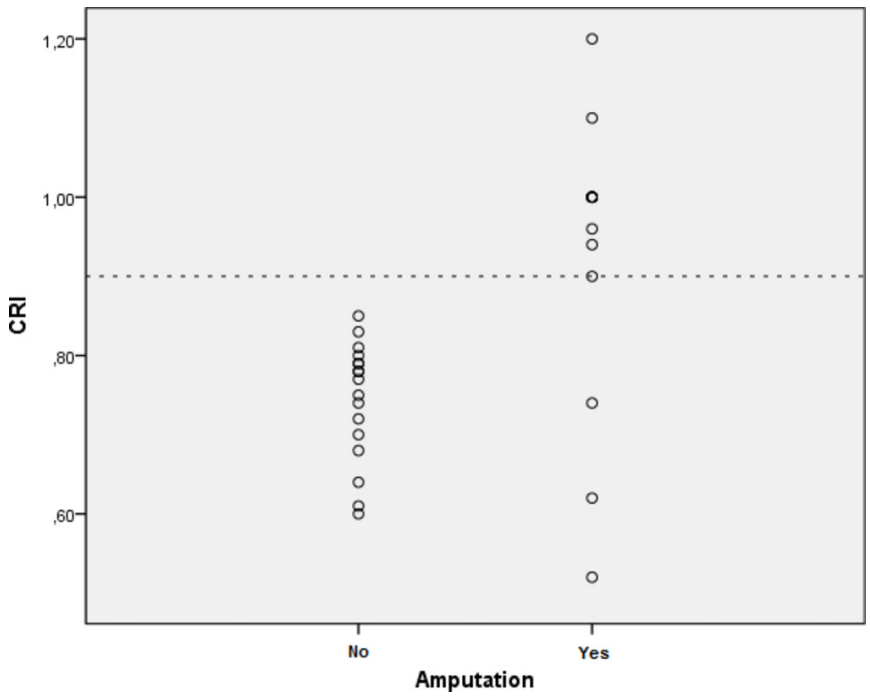

Figure 2 Scatter plot of $\mathrm{CRI}$ in patients with and without amputation. Fourteen patients did undergo an amputation. Most of these patients $(n=11$ ) had a CRI $\geq 0.9$ (of which six patients had a $\mathrm{CRI}=1.0$ ). Three patients had a $\mathrm{CRI}<0.9$ $(0.52,0.62$ and 0.74$)$. Seventeen patients did not undergo an amputation. These patients all had a $\mathrm{CRI}<0.9$ (of which two patients with $\mathrm{CRI}=0.78$ and two patients with $\mathrm{CRI}=0.79$ ). Dotted line indicates a potential cut-off value of $C R I=0.9$ to separate non-amputated patients with a positive flow reserve from the amputated patients. CRI, Capillary Resistance Index. 


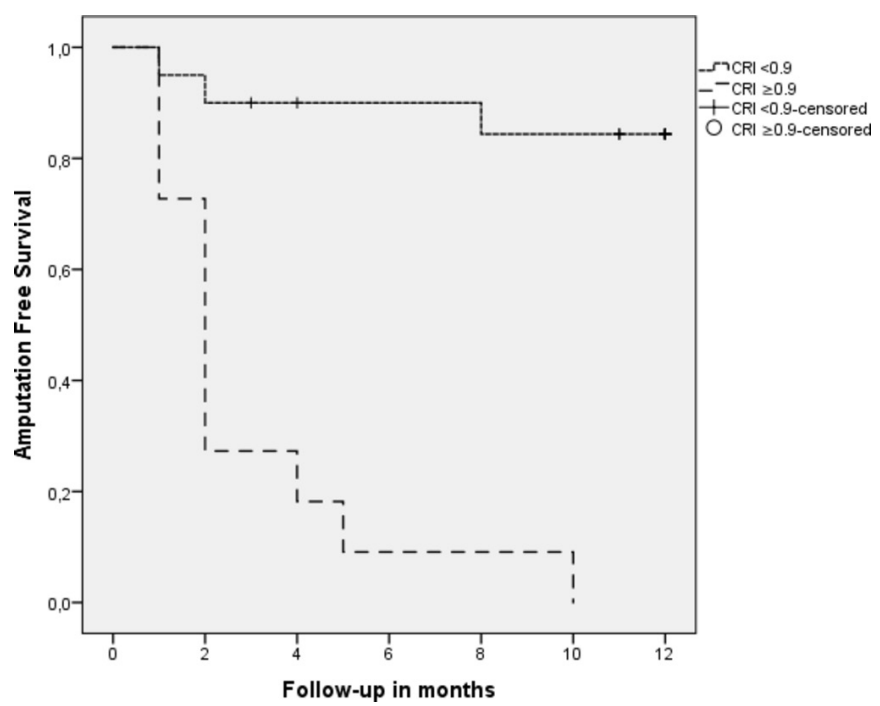

Figure 3 Kaplan-Meier curves for amputation-free survival in patients with $\mathrm{CRI}<0.9$ and with $\mathrm{CRI} \geq 0.9$ (log rank $\mathrm{p}<0.001$; HR 14.22 ; $95 \% \mathrm{Cl} 3.64$ to 55.51). Eleven patients with $C R I \geq 0.9$. Twenty patients with $C R I<0.9$. CRI, Capillary Resistance Index.

revascularization or bypass surgery. At 12 months' follow-up, 14 patients $(45 \%)$ had undergone a lower limb amputation. Nine patients died before 12 months' follow-up, two at 3 months, two at 4 months, one at respectively 7,8 and 10 months, and two at 11 months after intervention. Five of these patients had a lower limb amputation before they died. In all other patients the maximum follow-up was 12 months.

\section{CRI cut-off}

We calculated the CRI of all patients at 12 months. We compared the CRI of all patients whether or not they had undergone amputation. We made a scatter plot of the

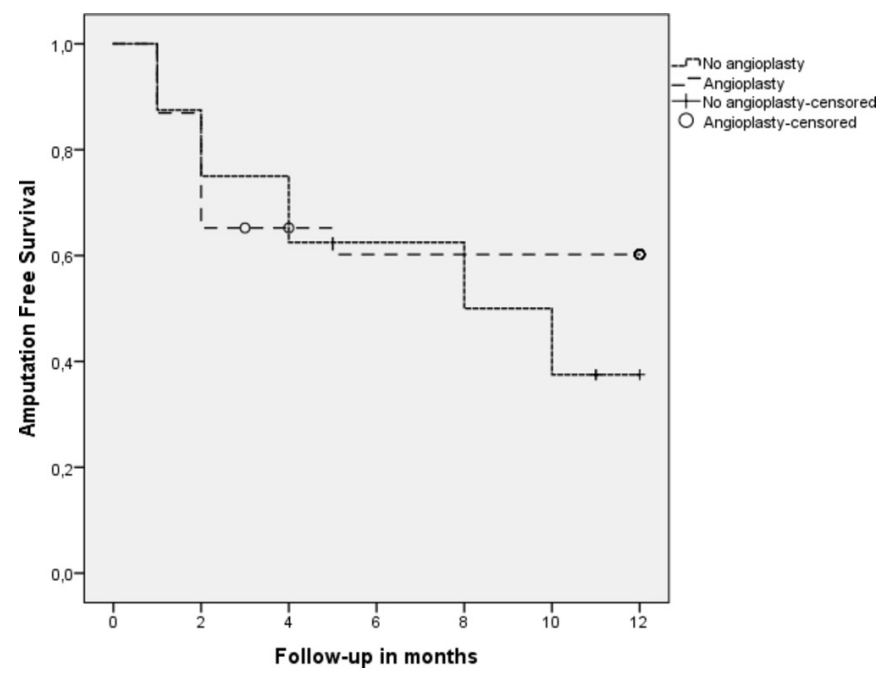

Figure 4 Kaplan-Meier curves of amputation-free survival in patients who did undergo angioplasty and in patients who did not undergo angioplasty (log rank $\mathrm{p}=0.407$; HR 0.65 ; $95 \% \mathrm{Cl} 0.22$ to 1.94). Twenty-three patients did undergo angioplasty, eight patients did not undergo angioplasty.

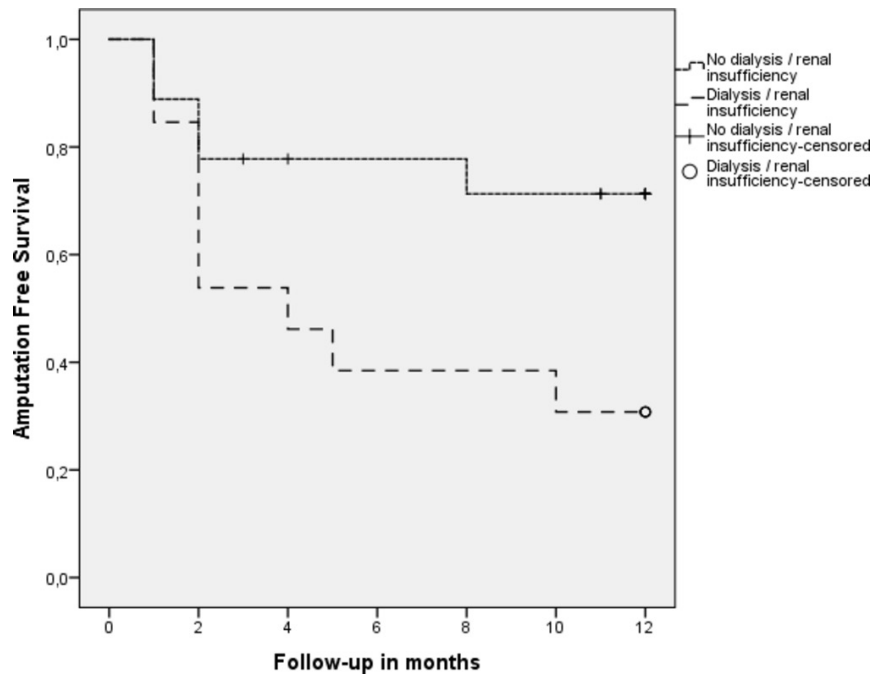

Figure 5 Kaplan-Meier curves of amputation-free survival in patients who had renal insufficiency or underwent dialysis and in patients with normal renal function (log rank $p=0.036$; HR 2.88; $95 \% \mathrm{Cl} 0.96$ to 8.64). Renal insufficiency=eGFR $<30$ $\mathrm{mL} / \mathrm{min}$. eGFR, estimated glomerular filtration rate.

CRI in patients with and without amputation and found a cut-off point of CRI $\geq 0.9$ (figure 2 ).

In the group of 20 patients with a $\mathrm{CRI}<0.9$, three patients $(15 \%)$ underwent amputation before 12 months. In the group of 11 patients with a CRI $\geq 0.9$, all patients $(100 \%)$ underwent amputation before 12 months. This results in a sensitivity of 0.79 (95\% CI 0.57 to 1.00$)$, specificity of 1.00 (95\% CI 1.00 to 1.00$)$, PPV of 1.00 (95\% CI 1.00 to $1.00)$ and NPV of 0.85 (95\% CI 0.69 to 1.00$)$.

\section{Amputation-free survival}

In this small first group of patients, the AFS showed a strong association ( $\log$ rank $\mathrm{p}<0.001$; HR 14.22; $95 \%$ CI 3.64 to 55.51) with the CRI (figure 3). The AFS was also calculated in patients who underwent angioplasty. Patients who did undergo an angioplasty had a slightly better AFS at 12 months, although this difference is not significant $(\log$ rank $\mathrm{p}=0.407$; HR $0.65 ; 95 \%$ CI 0.22 to 1.94). For details see figure 4 . There was no difference in AFS and type of diabetes (type 1 or 2 ; $\log$ rank $\mathrm{p}=0.699$; HR $0.81 ; 95 \%$ CI 0.25 to 2.58 ).

The AFS was worse in patients who had renal insufficiency (estimated glomerular filtration rate $<30 \mathrm{~mL}$ / min) or underwent dialysis ( $\log$ rank $\mathrm{p}=0.036$; HR 2.88; 95\% CI 0.96 to 8.64) (figure 5).

Table 2 Crosstabof patients with renal insufficiency or dialysis versus $\mathrm{CRI}<0.9$ and $\mathrm{CRI} \geq 0.9(p=0.010)$

\begin{tabular}{llcl}
\hline & $\begin{array}{l}\text { No dialysis/no } \\
\text { renal insufficiency }\end{array}$ & $\begin{array}{l}\text { Dialysis/renal } \\
\text { insufficiency }\end{array}$ & Total \\
\hline $\mathrm{CRI}<0.9$ & 15 & 5 & 20 \\
$\mathrm{CRI} \geq 0.9$ & 3 & 8 & 11 \\
Total & 18 & 13 & 31 \\
\hline
\end{tabular}

CRI, Capillary Resistance Index. 
The number of patients with normal renal function was significantly higher in the $\mathrm{CRI}<0.9$ group $(\mathrm{p}=0.010)$ (table 2). Mortality was also calculated in relation to $\mathrm{CRI}<0.9$ or $\geq 0.9$, and showed no significant difference.

\section{DISCUSSION}

It is to be expected that in a selective study population of patients with diabetes with CLI and a non-healing neuroischemic ulcer the amputation rate is very high, as was shown in our results. In this small and selective study group the functionality of the sympathetic nervous system was shown to be a strong single variable with a high significance $(\log$ rank $\mathrm{p}<0.001)$ in predicting clinical outcome. The PPV for AFS of less than 12 months in this first series is $1.00(11 / 11,95 \%$ CI 1.00 to 1.00$)$ in patients with a CRI $\geq 0.9$. The NPV for AFS of more than 12 months was $0.85(3 / 20,95 \%$ CI 0.69 to 1.00$)$ with a $\mathrm{CRI}<0.9$.

Significantly more patients with renal insufficiency or on dialysis were seen in the group with a CRI $\geq 0.9$. In patients with CLI, inflow arteries become maximally vasodilated by vasoactive metabolites released from ischemic tissue to improve the flow. During CLI, the arteries become resistant to vasoconstrictive or vasodilating drugs, known as 'vasomotor paralysis'. ${ }^{26}$ However, we have seen that during local critical ischemia the sympathetic autoregulation system of the arterioles continues to be responsive to an alpha-blocker. Therefore, this test can only be used under local ischemic conditions where the arteries are already maximally dilated.

The important role played by the sympathetic nervous system for the hemodynamic autoregulation of the microcirculation and the pathophysiology of the diabetic foot ulcer was first suggested 30 years ago and the concept has never totally disappeared. ${ }^{13-16}$ 27-30 The autoregulation of the microcirculation is important in the protection of the capillaries from high blood pressure, during changes of posture and walking, for example. ${ }^{127-29}$ Permanently high blood pressure in the capillaries leads to a traumatic response of the cellular matrix of the capillaries with leaking and subsequent thickening of the basement membrane. ${ }^{92}$ Accumulation of extravascular matrix proteins with subsequent thickening of the basement membrane with local deposition of hyaline is a ubiquitous finding having been demonstrated in the microcirculation of many organs in patients with diabetes. ${ }^{30}$ The mechanism of autoregulation is located in the arterioles and directed by the sympathetic nervous system and the local alpha-adrenergic receptors on the vessel wall of the arterioles. In $30 \%-50 \%$ of patients with diabetes with neuropathy there is also a dysfunctional sympathetic nervous system in the foot. ${ }^{31}$ The extent to which the sympathetic nervous system has to be dysfunctional before the local autoregulation becomes dysfunctional is unknown. In an animal experiment, it was shown that denervation of the sympathetic nerve by means of lumbar sympathectomy will make the alpha-adrenergic receptors completely insensitive to an alpha-adrenergic blocker such as Tolazoline. ${ }^{21}$ We therefore postulated that the non-response of the sympathetic nervous system to Tolazoline is a strong predictor for a dysfunctionality and a strong predictor for early amputation. There is further evidence for this hypothesis. A study that took laser Doppler skin perfusion pressure as a parameter for flow changes also observed that no response after injection of the vasodilator Alprostadil (Abbott), which is a synthetic prostaglandin E1 analogue with alpha-adrenergic blocking activity, has a high predictive value for amputation in patient with CLI. ${ }^{18}$ Our observations challenge a long existing paradigm that revascularization is always the solution to healing a neuroischemic ulcer in patients with diabetes. In the past decade, there has been a strong focus on PAD as an important predictor of outcome in CLI, both in patients with diabetes and the non-diabetic patients, and there has been enormous increase in revascularization procedures worldwide. However, more than $20 \%$ of all patients with diabetes who undergo endovascular or surgical revascularization still require major amputation within 12 months. ${ }^{56}$ The high amputation rate in our study population could be due to patient selection, but also in real-world studies the outcome in all patients with diabetic foot syndrome is high. ${ }^{10}$

In addition, it has become clear that the sympathetic nervous system also plays an important role in the defence against infection by initiating the inflammatory reflex. ${ }^{32}$ Therefore, poor functionality of the sympathetic nervous system could also be an explanation for the often uncontrolled and rapid spread of infection in diabetic foot disease.

In conclusion we have shown that with the aid of perfusion angiography we now have a very simple and highly sensitive diagnostic test to investigate the functionality of the sympathetic nervous system in the foot in patients with a neuroischemic ulcer. By using this test we are able to predict the outcome more accurately. This test could potentially be used as a proxy test for microangiopathy. One of the advantages of this test is that it blends seamlessly with our current practice of endovascular treatment. The initial clinical impact of our results could be that in patients with diabetes with a CRI $>0.9$ and a remaining or worsening non-healing ulcer after revascularization, a second reintervention is probably in vain. Whereas in patients with a CRI $<0.9$ a reintervention might be useful. This might be a very cost-effective strategy, especially because this test does not add anything to the cost of the intervention as it is a software algorithm using the routinely acquired angiography data. There is also no extra patient burden as the data are acquired during a routine intervention by an interventionalist.

Although the outcome regarding AFS is highly significant, the limitation of this study is that it is still a small selective study population and that the analysis is based only on routine clinical data. Patients with severe gangrene (Wagner 5) are in our institution not 
considered primary candidates for endovascular revascularization. This might be a possible selection bias. Before this test can be used in daily clinical practice it should be evaluated in a larger prospective study.

Contributors SMS: performing the study, data analysis and writing, final approval. MN and MJWK: study design, revising the manuscript, final approval. SB: study design, statistical analysis, final approval. JAR: study design, writing, data interpretation, revising the manuscript, final approval.

Funding The authors have not declared a specific grant for this research from any funding agency in the public, commercial or not-for-profit sectors.

Competing interests None declared.

Patient consent Not required.

Provenance and peer review Not commissioned; externally peer reviewed.

Open access This is an open access article distributed in accordance with the Creative Commons Attribution Non Commercial (CC BY-NC 4.0) license, which permits others to distribute, remix, adapt, build upon this work non-commercially, and license their derivative works on different terms, provided the original work is properly cited, appropriate credit is given, any changes made indicated, and the use is non-commercial. See: http://creativecommons.org/licenses/by-nc/4.0

\section{REFERENCES}

1. Skrepnek GH, Mills JL, Armstrong DG. A diabetic emergency one million feet long: disparities and burdens of illness among diabetic foot ulcer cases within emergency departments in the United States, 2006-2010. PLoS One 2015;10:e0134914.

2. Brownrigg JR, Hinchliffe RJ, Apelqvist J, et al. Performance of prognostic markers in the prediction of wound healing or amputation among patients with foot ulcers in diabetes: a systematic review. Diabetes Metab Res Rev 2016;32:128-35.

3. Wang Z, Hasan R, Firwana B, et al. A systematic review and metaanalysis of tests to predict wound healing in diabetic foot. $J$ Vasc Surg 2016;63:29S-36.

4. Mills JL, Conte MS, Armstrong DG, et al. The Society for Vascular Surgery Lower Extremity Threatened Limb Classification System: risk stratification based on wound, ischemia, and foot infection (WIfI). J Vasc Surg 2014;59:220-34.

5. Faglia E, Clerici G, Clerissi J, et al. Long-term prognosis of diabetic patients with critical limb ischemia: a population-based cohort study. Diabetes Care 2009;32:822-7.

6. Simons JP, Goodney PP, Nolan BW, et al. Failure to achieve clinical improvement despite graft patency in patients undergoing infrainguinal lower extremity bypass for critical limb ischemia. $J$ Vasc Surg 2010;51:1419-24.

7. Malyar NM, Freisinger E, Meyborg M, et al. Amputations and mortality in in-hospital treated patients with peripheral artery disease and diabetic foot syndrome. J Diabetes Complications 2016;30:1117-22.

8. Humphries MD, Brunson A, Hedayati N, et al. Amputation risk in patients with diabetes mellitus and peripheral artery disease using statewide data. Ann Vasc Surg 2016;30:123-31.

9. Parving HH, Viberti GC, Keen $\mathrm{H}$, et al. Hemodynamic factors in the genesis of diabetic microangiopathy. Metabolism 1983;32:943-9.

10. Flynn MD, Tooke JE. Aetiology of diabetic foot ulceration: a role for the microcirculation? Diabet Med 1992;9:320-9.
11. Fagrell B, Jörneskog G, Intaglietta M. Disturbed microvascular reactivity and shunting - a major cause for diabetic complications. Vasc Med 1999;4:125-7.

12. Flynn MD, Tooke JE. Diabetic neuropathy and the microcirculation. Diabet Med 1995;12:298-301.

13. Ahmed ME, Delbridge L, Le Quesne LP. The role of autonomic neuropathy in diabetic foot ulceration. J Neurol Neurosurg Psychiatry 1986;49:1002-6.

14. McFadden JP, Corrall RJ, O'Brien IA. Autonomic and sensory nerve function in diabetic foot ulceration. Clin Exp Dermatol 1991;16:193-6.

15. Dellantonio R, Paladini D, Cinti A, et al. Autonomic diabetic neuropathy: skin sympathetic response and neurovegetative cardiovascular functionality tests. Funct Neurol 1992;7:115-20.

16. Cacciatori V, Dellera A, Bellavere F, et al. Comparative assessment of peripheral sympathetic function by postural vasoconstriction arteriolar reflex and sympathetic skin response in NIDDM patients. Am J Med 1997;102:365-70.

17. Zimny S, Dessel F, Ehren M, et al. Early detection of microcirculatory impairment in diabetic patients with foot at risk. Diabetes Care 2001;24:1810-4.

18. Muto A, Kondo Y, Pimiento JM, et al. Vasodilator response correlates with outcome in chronic critical limb ischemia. J Surg Res 2010;161:156-61.

19. Cuypers $Y$, Steels $P$. Relationship between the radioisotope test using 131/-labelled serum albumin and plethysmographic blood flow measurements in the study of the circulation in the foot. Angiology 1981;32:742-51.

20. Reekers JA, Koelemay MJ, Marquering HA, et al. Functional imaging of the foot with perfusion angiography in critical limb ischemia. Cardiovasc Intervent Radiol 2016;39:183-9.

21. Dalessandri KM, Lantz BM, Palmaz JC. The effect of lumbar sympathectomy on postsynaptic vascular smooth muscle response in the lower limb in dogs. Cardiovasc Intervent Radiol 1988;11:82-5.

22. Darcy MD. Lower-extremity arteriography: current approach and techniques. Radiology 1991;178:615-21.

23. Benoit E, O'Donnell TF, Kitsios GD, et al. Improved amputationfree survival in unreconstructable critical limb ischemia and its implications for clinical trial design and quality measurement. $J$ Vasc Surg 2012;55:781-9.

24. van Battum P, Schaper N, Prompers L, et al. Differences in minor amputation rate in diabetic foot disease throughout Europe are in part explained by differences in disease severity at presentation. Diabet Med 2011;28:199-205.

25. Douglas G. Altman. Practical statistics for medical research, 1999: 230.

26. Cardinal TR, Struthers KR, Kesler TJ, et al. Chronic hindlimb ischemia impairs functional vasodilation and vascular reactivity in mouse feed arteries. Front Physiol 2011;2:91.

27. Kastrup J, Nørgaard T, Parving $\mathrm{HH}$, et al. Impaired autoregulation of blood flow in subcutaneous tissue of long-term type 1 (insulindependent) diabetic patients with microangiopathy: an index of arteriolar dysfunction. Diabetologia 1985;28:711-7.

28. Flynn MD, Tooke JE. Microcirculation and the diabetic foot. Vasc Med Rev 1990;vmr-1:121-38.

29. Tooke JE. Microvasculature in diabetes. Cardiovasc Res 1996;32:764-71.

30. Williamson JR, Kilo C. Capillary basement membranes in diabetes. Diabetes 1983;32:96-100.

31. Watkins PJ, Edmonds ME. Sympathetic nerve failure in diabetes. Diabetologia 1983;25:73-7.

32. Pongratz G, Straub RH. The sympathetic nervous response in inflammation. Arthritis Res Ther 2014; 16:504. 